\title{
Nomadisme et semi-nomadisme au Maroc
}

Mohamed Aït Hamza

\section{OpenEdition}

Journals

Édition électronique

URL : https://journals.openedition.org/encyclopedieberbere/2752

DOI : 10.4000/encyclopedieberbere. 2752

ISSN : 2262-7197

\section{Éditeur}

Peeters Publishers

\section{Édition imprimée}

Date de publication : 5 octobre 2012

Pagination : 5602-5609

ISBN : 978-90-429-2718-6

ISSN : 1015-7344

\section{Référence électronique}

Mohamed Ait Hamza, « Nomadisme et semi-nomadisme au Maroc », Encyclopédie berbère [En ligne], 34 | 2012, document N66, mis en ligne le 15 décembre 2020, consulté le 17 février 2022. URL : http:// journals.openedition.org/encyclopedieberbere/2752 ; DOI : https://doi.org/10.4000/ encyclopedieberbere. 2752

Ce document a été généré automatiquement le 17 février 2022.

(c) Tous droits réservés 


\title{
Nomadisme et semi-nomadisme au
}

\section{Maroc}

\author{
Mohamed Aït Hamza
}

1 Evoquer, aujourd'hui le nomade au Maroc peut sembler être un anachronisme. Le nomade défini comme personne mobile sans habitation fixe, n'existe quasiment plus. Dans le cas marocain, on parlera plutôt du semi-nomade. Ces populations constituent une catégorie rarement évoquée, sauf pour parler de ses conflits autour des points d'eau ou des parcours ou encore pour évoquer la dégradation des ressources dont elle est soupçonnée être la cause. L'éleveur transhumant est cependant le principal fournisseur de viande, de laine et de cuir. Il est, malgré tout ce qu'on dit de lui, par de bonnes pratiques, le vrai protecteur des ressources naturelles.

2 L'image négative portée sur cette communauté, fait que l'appareil statistique n'en fait que rarement état. Les seuls chiffres dont on dispose sont donnés par des recensements généraux. Ils sont 13.475 ménages lors du recensement 1982 et seulement 12.636 ménages en 1994, hormis les provinces sahariennes! (Yessef et Ait Hamza 2009)

3 Eu égard à leur mode de vie, à leur culture, l'image construite autour de cette population, n'est généralement qu'une perception plus au moins tronquée de la réalité. La présente note tente, à travers de nombreux écrits et des travaux de terrain récents (voir bibliographie) de faire le point sur l'évolution de ce phénomène. 


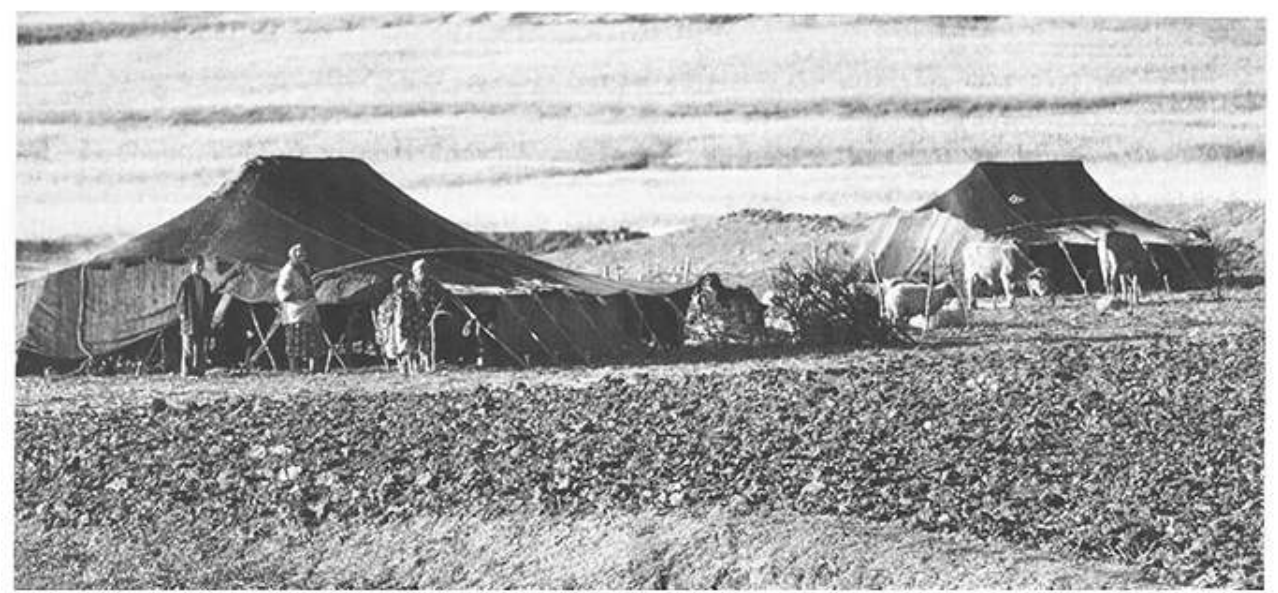

Photo 1. AXam (tente des imazighen), Aghbala n AYt-SUKhman

Cliché M. Peyron.

\section{Le nomade et son territoire au Maroc}

4 L'image relativement négative des nomades reflète grosso modo la relation que ceux-ci avaient avec le Makhzen central. En fait, ces communautés «sans adresse fixe » ne se soumettent que rarement aux limites territoriales et à la réglementation qu'impose l'administration centrale. Ainsi, les nomades, sans remettre en cause le pouvoir central, n'hésitent pas à transgresser les limites qu'il impose et à menacer l'ordre établi chaque fois que le besoins se fait sentir.

Dès le XVII-XVIII ${ }^{e}$ siècle, les rois du Maroc, face à la descente des montagnards vers les plaines et les capitales impériales, ont senti le besoin de bâtir de nombreuses kasbahs et d'implanter de nombreuses tribus guich-s sur leurs routes. Le Bled s-Siba dont parlaient les historiens pour décrire la dissidence au XIX ${ }^{\mathrm{e}}$ siècle n'est autre qu'un refus de se plier aux exigences financières du pouvoir central. La France coloniale, pour châtier la résistance des montagnards, les prive de leur droit à la forêt et aux terres de parcours (domanialisation des eaux, des forêts et mainmise sur les terres collectives).

6 Aujourd'hui, face aux problèmes que posent la montagne et ses populations (environnement, emploi et autres besoins sociaux), il est tout à fait légitime de se poser des questions sur la relation qui peut exister entre le mode de vie mobile des populations et la préservation de l'environnement.

$7 \mathrm{Au}$ Maroc, malgré leur fragilité, les conditions naturelles se caractérisent par une très grande diversité. La recherche de la complémentarité des territoires et des finages est une forme intelligente d'adaptation à l'irrégularité des précipitations et des aléas. Le nomade, au vu de son expérience, adapte ses itinéraires de transhumance aux impératifs que lui imposent le relief et le climat. Il en résulte, pour chaque communauté, un finage, idéal composé de différents terroirs permettant l'exercice de ses activités, avec une relative stabilité saisonnière et annuelle.

8 La recherche de cette complémentarité entre les sommets de montagne cléments et herbacés en été et les altitudes basses à températures douces en hiver, oriente le mouvement du cheptel. Ce schéma donne à la végétation un moment de répit et de repos nécessaire à la régénération. Cette contrainte impose à chaque communauté la 
conquête de ces espaces soit par le fusil, soit en recourant aux alliances (leff-s) interfractions ou intertribales. Il en résulte un maillage tribal très compliqué. A titre d'exemple, la tribu des Ait Sedrate, en s'installant sur trois sections distinctes du bassin du Dadès (amont, moyen et aval) s'est assurée la maîtrise, mais pas sans conflits, de l'espace nécessaire à l'entretien d'un troupeau durant toutes les saisons. Les conflits autour des points d'eau, des parcours et des passages forment la trame de l'histoire locale. Avec l'avènement de l'administration moderne, l'activité d'élevage et, par conséquent la vie des éleveurs mobiles, a connu de profondes mutations. Pour mieux saisir ces mutations, quatre bassins écologiques de transhumance ont été différentiés (Yessef et Aït Hamza 2009).

\section{Les différents bassins du nomadisme}

\section{Les nomades chameliers du Sahara}

Zone très étendue entre l'embouchure du Dra et la frontière maroco-mauritanienne, le Sahara marocain est connu par l'importance de ses cheptels, surtout de camelins. Activité basée essentiellement sur de longs mouvements, le nomadisme est l'œuvre de grandes tribus : Rguibat, Ouled Dlim, Ouled Bousbaâ, Zerguiyne, Ait Oussa, Tekna.... Outre l'élevage, ces tribus pratiquent un commerce transfrontalier et un artisanat sommaire (Dahmane 2004, 2006). Le nomadisme constitue le genre de vie dominant pour tous les membres de la tribu. Les moments d'arrêt pour pratiquer des cultures pluviales sont rarissimes (culture dans les grair-s). Le pourcentage des éleveurs mobiles par rapport au nombre total des éleveurs atteint le niveau le plus élevé. Le pourcentage des troupeaux qui pratiquent une mobilité coutumière atteint $75,6 \%$ et celui des troupeaux qui effectuent des déplacements hors des territoires coutumiers est de $24.4 \%$.

10 A partir de 1975, pour des raisons de sécurité, l'Etat a encouragé la sédentarisation et l'urbanisation en installant des équipements de base dans des points focaux (Laayoune, Boujdour, Dakhla, Es-Smara, Tarfaya, Tantan). Ces villes créées pour restructurer l'espace, concentrent déjà plus de $94 \%$ des habitants (RGPH, 2004). L'équilibre économique régional, jadis fondé sur le nomadisme, s'oriente désormais vers la pêche, le commerce et l'exploitation minière. L'élevage est de plus en plus pratiqué par des intermédiaires gérants, alors que les propriétaires, installés en ville, s'adonnent au commerce et à la spéculation.

\section{Le Haut-Atlas et ses bordures, espace des semi-nomades}

11 Le Haut-Atlas et ses bordures constituent un espace traditionnellement exploité par de grandes confédérations de tribus: Aït Atta, Aït Yafelmane, Aït Sedrate, Glaoua, Ghojdama, Mgouna, Imaghrane, Aït Isha, Aït Sokhmane... Ces communautés, en grande partie sédentaires, pratiquaient la transhumance selon des itinéraires verticaux orientés Sud/Nord. En été, les troupeaux sont menés sur les agdal-s ${ }^{1}$ d'altitude, alors $^{\prime}$ qu'en hiver, celles-ci se rapprochaient des oasis de piémont. La pratique de la mise en défens temporaire des parcours d'été (agdal) constitue la spécificité de cette aire. Les tribus concernées par la transhumance ont développé une réglementation stricte qui 
préservait les droits de chaque éleveur, mais et surtout, assurait la protection de la nature contre la surexploitation des ressources.

Avec l'installation de l'administration moderne, l'effritement de la jmaa traditionnelle, la sédentarisation accrue, l'ouverture, la dégradation deviennent la règle (Ait Hamza, 2005 ; Ouhajou, 2006). Le pourcentage du cheptel qui pratique encore la mobilité atteint $49,6 \%$, mais celui des éleveurs ne dépassent guère $37,2 \%$ dont $17,9 \%$ hors de leur territoire coutumier.

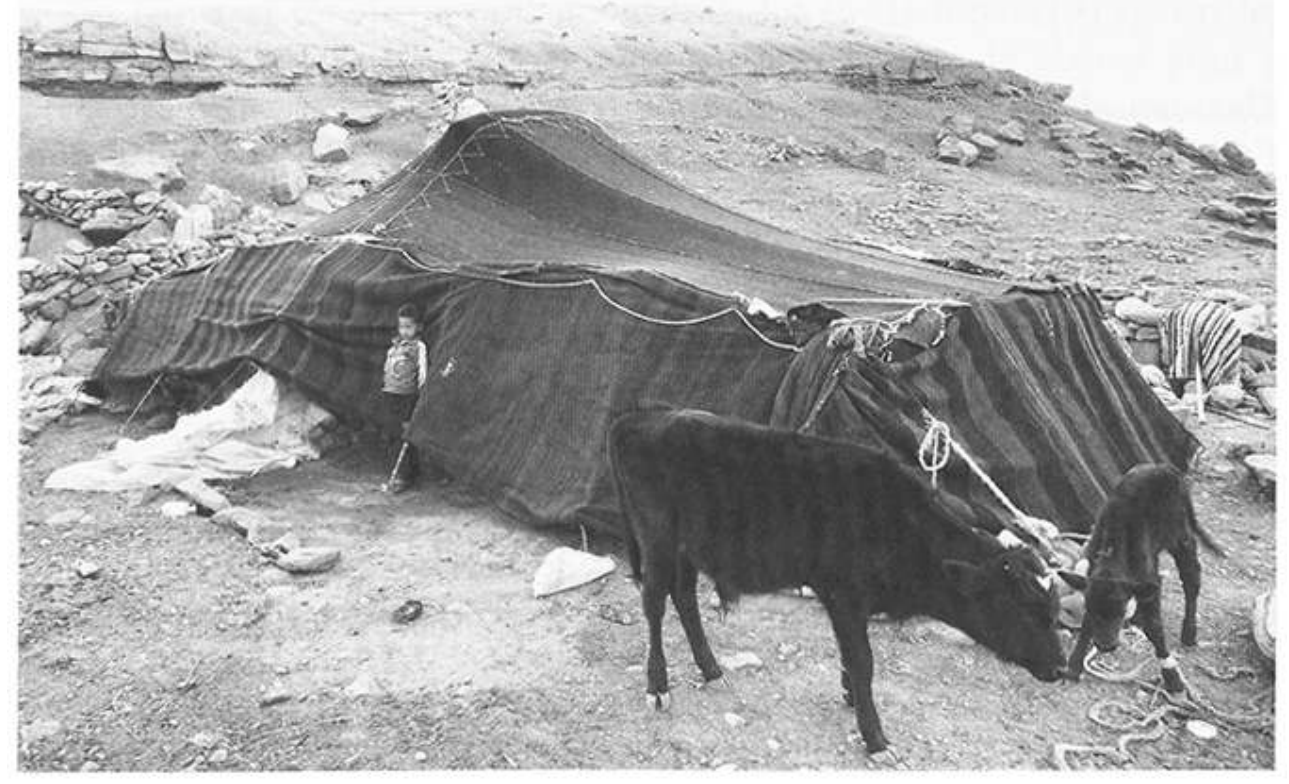

Photo 2. AU Pied du haut ATLAS ORIental (MIDelt), SEPTEMBre 2001.

Cliché Ali Swirti.

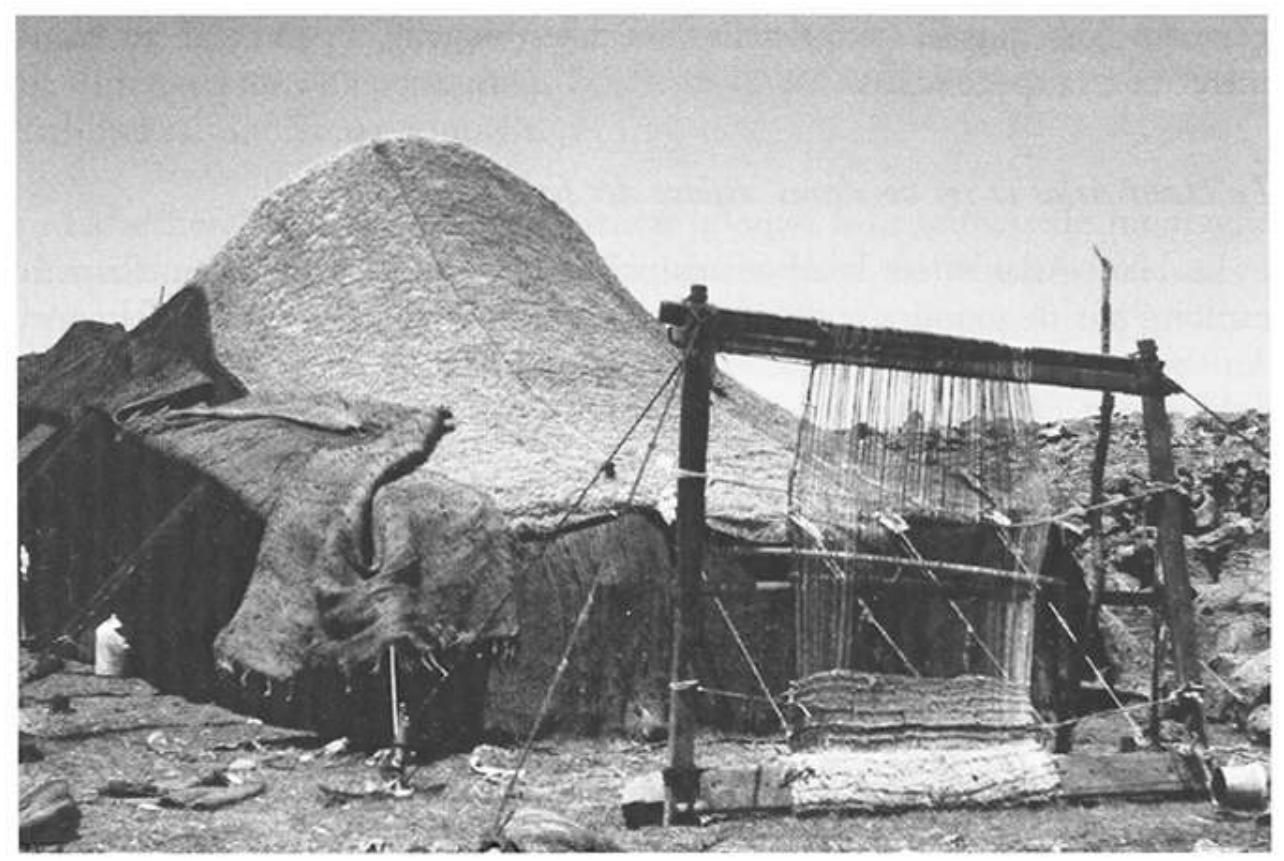

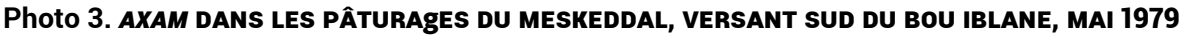

Cliché Hillary Nias. 


\section{Une nouvelle ère pour les transhumants des Hauts-Plateaux de l'Orientale}

Eu égard à son relief de moyennes altitudes, à son aridité relative, la zone de l'Orientale forme un espace d'élevage à mouvements d'ampleur restreint (El Harrach 1997). La transhumance se pratique entre jbel Grouz au sud et le Horst de Jerada au nord. Cette mobilité est essentiellement favorisée par la complémentarité des vastes faciès pastoraux steppiques dominés par l'alfa et l'armoise. La confédération de tribus la plus imposante est celle de Beni-Guil avec ses multiples fractions, suivie par celle des Laamour. Les effectifs du petit bétail, concernés par la mobilité représentent 49,3\%. Le pourcentage des éleveurs qui pratiquent l'élevage mobile est d'environ $36,9 \%$ dont $19,6 \%$ hors du territoire coutumier. Très touchés par la sécheresse et la fermeture de la frontière algéro-marocaine, cette zone a connu une intervention majeure de l'Etat qui visait à améliorer les parcours. Ce projet, en se basant sur l'encouragement des coopératives pastorales, le creusement des puits, l'achat des fourrages et l'amélioration de la race locale, a beaucoup stimulé l'intensification de l'élevage et par conséquent la sédentarisation (Ait Hamza 2011). Il a surtout profité aux grands éleveurs d'ovins installés à Tandrara, Aïn-Beni-Mathar et Bouarfa.

\section{Les semi-nomades du Moyen-Atlas et l'intensification de l'agriculture}

Le Moyen Atlas se caractérise par une régression dramatique de l'élevage mobile. La mainmise du colonisateur sur les terrains de parcours des Ayt Mgild, dans l'Azaghar (Beaudet 1969) et la récente mise en culture de cette montagne notamment dans sa partie occidentale (El Hajeb, Azrou, Imouzzer, Ifrane, Guigou...) en y introduisant les céréales, le maraîchage, l'arboriculture fruitière..., l'élevage moderne intensif ou semiintensif, a entraîné une sédentarisation accélérée. Le tourisme et l'armée ont aussi arraché à l'élevage une main-d'œuvre nécessaire à son maintien. Le pourcentage des troupeaux encore concernés par la mobilité n'est que de l'ordre de $37,3 \%$ !, alors que le pourcentage des éleveurs qui pratiquent l'élevage mobile ne dépasse guère $35.2 \% \mathrm{du}$ total !.

\section{Conclusion}

Il serait aisé de conclure que l'on assiste à la fin du nomadisme et du semi-nomadisme au Maroc, du moins dans leur forme traditionnelle. Les raisons de ce processus de régression sont multiples, même si leurs ampleurs et leurs impacts diffèrent selon les bassins écologiques de transhumance.

16 En fait, très tôt, la promulgation de la loi sur la forêt (1917), sur les terres collectives (1919), la mainmise sur les parcours de plaines pour y créer des périmètres irrigués, ont entamé un processus de régression quasi irréversible.

17 Avec l'indépendance et les nouvelles orientations de l'Etat, l'importance accordée à l'agriculture irriguée et à l'aménagement des villes ont réduit encore les espaces pastoraux et les zones bour.

18 L'exode déjà enclenché dès la fin du XIX ${ }^{\mathrm{e}}$ siècle s'est amplifié et les effets de l'ouverture, de la scolarisation rurale, du contact avec les villes et les médias, se sont intensifiés 
avec l'explosion démographique. Le nomade jadis isolé avec ses bêtes face à la nature, aspire, aujourd'hui, a profiter des nouveaux éléments de confort qu'offre la vie moderne (camion, téléphone, médicament, école, électricité...). Les déplacements s'appuient dorénavant sur ces nouveaux moyens et emmènent les nomades très loin. La location des parcours, l'achat des fourrages, la recherche de l'adéquation entre la production et la demande caractérisent la nouvelle situation. Les institutions traditionnelles de gestion de la transhumance, comme la nouvelle administration, ont beaucoup de mal à suivre l'évolution. Autant de prémices qui nous font dire que la transhumance n'est peut-être pas perdue totalement, même si les jeunes et les femmes refusent de plus en plus ce mode d'élevage.

\section{ZONES HOMOGENES D'ELEVAGE MOBILE AU MAROC}

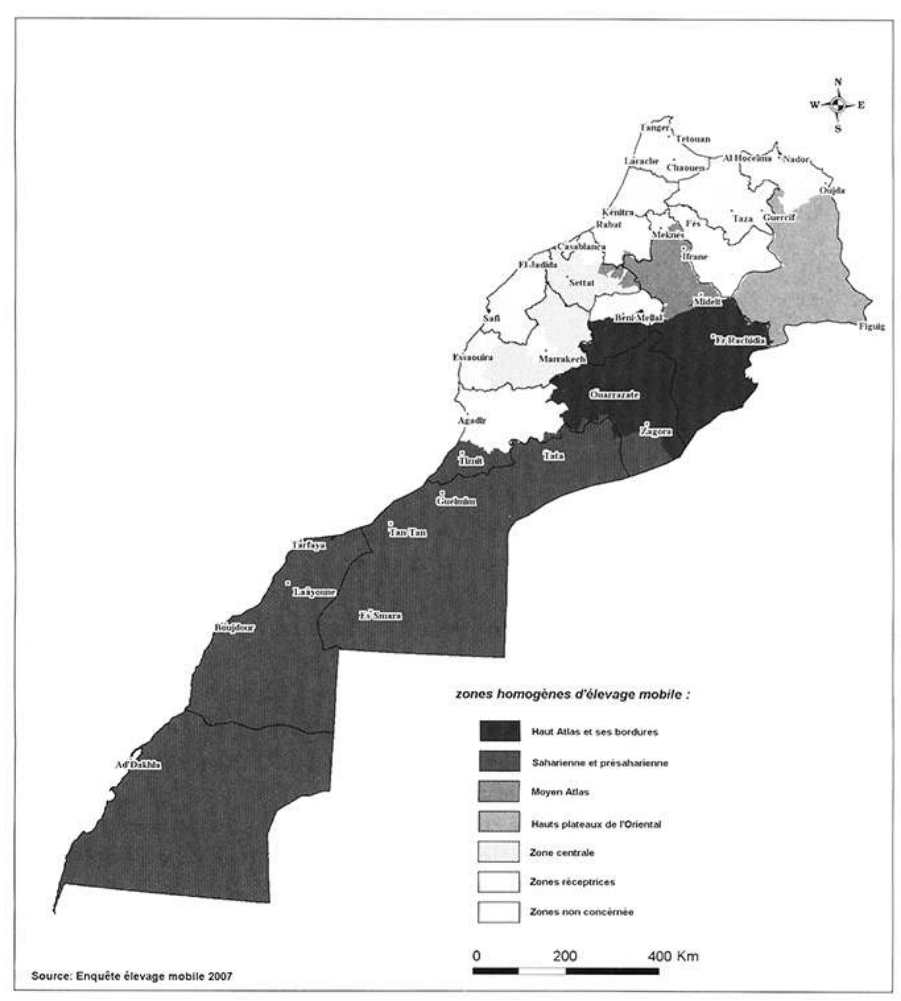

\section{BIBLIOGRAPHIE}

AIT HAMZA M., 2008 - « Nomadisme et transhumance : approche méthodologique », In Vers une stratégie de planification du développement territorial dans le monde arabe Actes de la $4^{\mathrm{e}}$ rencontre des Géographes arabes, Casablanca, ANAGEM, p. 663-693.

AIT HAMZA M., 2005 - «Crise de la montagne et forme d'adaptation (Maroc) », In Pour une nouvelle perception des montagnes marocaines, Ait Hamza M., et Popp H. Eds., Rabat, FLSH (Série Colloques et Séminaires, $\mathrm{n}^{\circ}$ 119). p. 17-24. 
AIT HAMZA M., 2011 - «Les coopératives de pasteurs : une expérience et des leçons », In Produits agricoles, touristiques et développement local, Kerzazi M., Ait Hamza, et Al Assaad M. (éd), Rabat, 2007 (Acte du Colloque organisé par l'ANAGEM/UGI), p. 57-68.

BEAUDET G., 1969 - « Les Ayt Mgild du nord, étude géographique de l'évolution récente d'une confédération semi-nomade ». RGM, 15, pp. 3-80, Voir résumé : « Mgild (Ayt Mgild)», In Encyclopédie Berbère, XXXII, 2010, p. 4977-4983.

BENCHERIFA A., 1995 - « Processus de sédentarisation et risque de désertification : impact environnemental de l'évolution récente du nomadisme pastoral dans les Hauts Plateaux du Maroc ». In Afrique du Nord face aux menaces écologiques, Rabat, FLSH (Série Colloques et Séminaires, $n^{\circ}$ 50), p. 79-97.

BOURBOUZE A., 1982 - L'élevage dans la montagne marocaine: Organisation de l'espace et utilisation des parcours par les éleveurs du Haut Atlas, Thèse de Docteur-Ingénieur, Paris, INA, 345 p. + pl.

CELERIER J. 1927 - « La transhumance dans le Moyen Atlas », Hespéris, t. 7.

CHICHE J., 2003 - Les conflits pastoraux sur le versant sud du Haut-Atlas. Projet CBTHA. ORMVA

Ouarzazte/PNUD, (Rapport) $302 \mathrm{p}$.

COUVREUR G., 1968 - « La vie pastorale dans le Haut Atlas central ». Revue de Géographie du Maroc, $\mathrm{n}^{\circ} 13$, p. 3-54.

DAHMANE M., 2004 - La transhumance et la sédentarisation : étude socio-historique de la tribu des Bani Bou Sbaa, Rabat, FLSH, Thèse en Sociologie, 2 t. (en arabe)

DAHMANE M., 2006 - La transhumance et la sédentarisation : Région de Séguia El Hamra et Oued Eddahab, Rabat, Imp. Kaoutar (en arabe).

DRESCH J., 1941 - Documents sur les genres de vie dans le massif central du Grand Atlas. Publications IHEM, t. XXXV, Tours. Arrault et Cie.

HAMMOUDDOU M. 1996 - L'élevage pastoral chez les Mgouna : étude des parcours et des systèmes d'élevage. ORMVA Ouarzazate (Rapport) $49 \mathrm{p}$.

HANAFI A., 1984 - La mutation économico-sociale de la tribu semi-nomade Erklaouen (Moyen-Atlas), Univ. Toulouse-Le Mirail. Thèse de Géographie, 310 p.

HARRACH M., 1997 - Organisation coopérative de gestion de l'espace et dynamique du système agropastoral dans l'oriental du Maroc, Montpellier, CHEAM/IAM Master of Science, 139 p., annexes

JENNAN L. 2004 - Le Moyen Atlas central et ses bordures : mutations récentes et dynamiques rurales, Edition El Jawahir, 706 p.

ouHAJOu L., ZAINABi A. T. et El MAHDAD H., 2006 - « Le nomadisme en déclin : le cas du Dra Moyen », Projet d'Atlas des Aires Protégées du Maroc. HCE-FLCD, 10 p. (rapport non publié).

MAHDI M., 2002 - Du nomadisme aux nouvelles formes d'élevage, In Mutations sociale et réorganisation des espaces steppiques, Casablanca, Ed. Mahdi, p. 65-90.

RACHIK H., 2000 - « Comment rester nomade ?» Afrique - Orient, Casablanca.

TOZY M., 2002 - « Des tribus aux coopératives ethno-lignagères », In Mutations sociales et réorganisation des espaces steppiques, Casablanca, Ed. Mahdi, p. 17-38

YESSEF M. et AIT HAMZA M., 2009 - Etude sur les structures et les tendances de la transhumance au Maroc. Projet de Conservation de la Biodiversité par la Transhumance dans le versant sud du Haut-Atlas (CBTHA). ORMVA Ouarzazate / PNUD (rapport inédit), 123 p. 
ZAINABI A.T., 1989 - « Vers une disparition rapide du nomadisme au Sahara marocain : le cas du Draa Moyen ", Le nomade, l'oasis et la ville. Fascicule de recherche $\mathrm{n}^{\circ} 20$ (Tours, URBAMA), p. 49-62.

\section{NOTES}

1. Agdal, terme pan-berbère: "pâturage, prairie mise en défens ", du verbe gdel, "protéger, mettre à l'abri, interdire, réserver... ", $C f$. K. Naït-Zerrad, Dictionnaire des racines berbères, III, Louvain/Paris, Peeters, 2002, p. 729-732. NDLR.

INDEX

Mots-clés : Commerce, Economie, Ethnologie, Maroc, Maroc central (Zone tamazight), Sahara 